\title{
A Millimeter and Submillimeter Kinetic Inductance Detector Camera
}

\author{
J. Schlaerth • A. Vayonakis · P. Day $\cdot$ J. Glenn • \\ J. Gao • S. Golwala • S. Kumar • H. LeDuc • \\ B. Mazin · J. Vaillancourt · J. Zmuidzinas
}

Received: 23 July 2007 / Accepted: 5 October 2007 / Published online: 25 January 2008

(C) Springer Science+Business Media, LLC 2008

\begin{abstract}
We present results from a demonstration camera using Microwave Kinetic Inductance Detectors (MKIDs) (Day et al. in Nature 425, 817-821, 2003) at the Caltech Submillimeter Observatory. The focal plane consists of 16 two-color (240 and $350 \mathrm{GHz}$ ) pixels. Each pixel is a phased-array of slot dipole antenna whose output power is coupled to MKIDs via in-line color-defining bandpass filters. A prototype software-defined radio system was used to read out up to four MKIDs simultaneously. We obtained maps of Jupiter, Saturn, and G34.3 and demonstrated sensitivities of approximately $1 \mathrm{Jy} \mathrm{s}^{1 / 2}$ and $10 \mathrm{Jy} \mathrm{s} \mathrm{s}^{1 / 2}$ in the two bands, respectively, limited by detector noise due to a low-efficiency optical train. We anticipate that a second engineering run in 2008 with a 36-element, 4-color array and an optimized optical train will be background limited at 240,270,350, and $400 \mathrm{GHz}$. We are undertaking the construction of a full-size MKID camera with 576 four-color spatial pixels and using 2304 MKIDs readout by an expanded software-defined radio system.
\end{abstract}

Keywords Radio telescopes and instrumentation · Heterodyne receivers · Superconducting infrared $\cdot$ Submillimeter and millimeter wave detectors

PACS 95.55.Jz $\cdot 85.25 . \mathrm{Pb}$

J. Schlaerth $(\bowtie) \cdot$ J. Glenn

Center for Astrophysics and Space Astronomy, University of Colorado, 593 UCB, Boulder, CO, USA

e-mail: James.Schlaerth@Colorado.edu

A. Vayonakis $\cdot$ J. Gao $\cdot$ S. Golwala $\cdot$ S. Kumar $\cdot$ J. Vaillancourt $\cdot$ J. Zmuidzinas

California Institute of Technology, 1200 E. California Blvd., M/S: 320-47, Pasadena, CA 91125,

USA

P. Day $\cdot$ H. LeDuc $\cdot$ B. Mazin

NASA Jet Propulsion Laboratory, 4800 Oak Grove Drive, M/S: 169-506, Pasadena, CA 91109, USA 


\section{Introduction to MKIDs}

Although superconductors exhibit zero DC resistance, there still exists a nonzero surface impedance to AC currents. This impedance is composed of a real resistive part, and an imaginary inductive part. Absorbed radiation will break Cooper pairs, changing its surface impedance. This change in impedance, which is composed of a real resistive part and an imaginary inductive part, will change the resonant frequency of a superconducting resonant circuit.

Microwave Kinetic Inductance Detectors (MKIDs) [1] are essentially high-Q superconducting resonant circuits coupled capacitively to a feedline. A change in resonant frequency due to absorption of radiation can be detected by sending a probe signal on-resonance through the feedline; the probe signal will change in its imaginary (phase) and real (amplitude) components as the surface impedance changes. For small enough changes in resonant frequency, this response is linear, and thus useful for astronomical observations. In practice, many different resonators at slightly different frequencies can be coupled to the same feedline, allowing for natural frequency multiplexing with negligible crosstalk. A cryogenic HEMT amplifies the signal with little noise after the device, allowing a high precision room temperature readout of the transmitted amplitude and phase.

We demonstrated the astronomical viability of this type of detector by using the detectors in a demonstration camera (DemoCam) at the Caltech Submillimeter Observatory (CSO) in Mauna Kea, Hawaii.

\section{Focal Plane Design}

The focal plane of the device uses lithographed phased arrays of slot antennas to couple the radiation to the resonators. In this design, 16 slot antennas are coadded inphase to produce a signal in a microstrip feedline. The slot antennas define the beam shape in the far field, and can be approximated by a uniformly illuminated square, $3.3 \mathrm{~mm}$ on a side, on the focal plane. The coupled radiation is then passed through inline lumped-element bandpass filters and coupled to a coplanar waveguide resonator, with a $6 \mu \mathrm{m}$ center strip and $2 \mu \mathrm{m}$ slots, and approximately $4 \mathrm{~mm}$ long. Thus, there is no need for band-defining optical filters; the necessary bandpasses were achieved solely by on-chip filters designed by the MKID group. The focal plane containing the MKID device, a $4 \times 4$ pattern of such antennas with resonators coupling to a meandered feedline, was cooled using a sorption fridge to approximately $230 \mathrm{mK}$, well below the $T_{c}$ of Aluminum.

The DemoCam was designed for simultaneous two-color operation as a precursor to future multicolor cameras. The antennas coupled to bands centered at $240 \mathrm{GHz}$ and $350 \mathrm{GHz}$, corresponding to the atmospheric windows of low attenuation (Fig. 1). The resonant frequencies of the detectors were set at $10 \mathrm{MHz}$ apart, beginning at $7.5 \mathrm{GHz}$.

\section{Instrument Design and Readout}

In order to couple the radiation to the detectors, we followed the same optical focusing procedure used by Bolocam [2]. This involves using a series of flat and elliptical 
Fig. 1 (Color on line) Fourier Transform Spectrometer data from the two bandpasses used in the DemoCam superposed on a plot of atmospheric transmission. The non-uniformity in the bandpasses is believed to be caused by reflections in the optical filter stack, though tests are ongoing

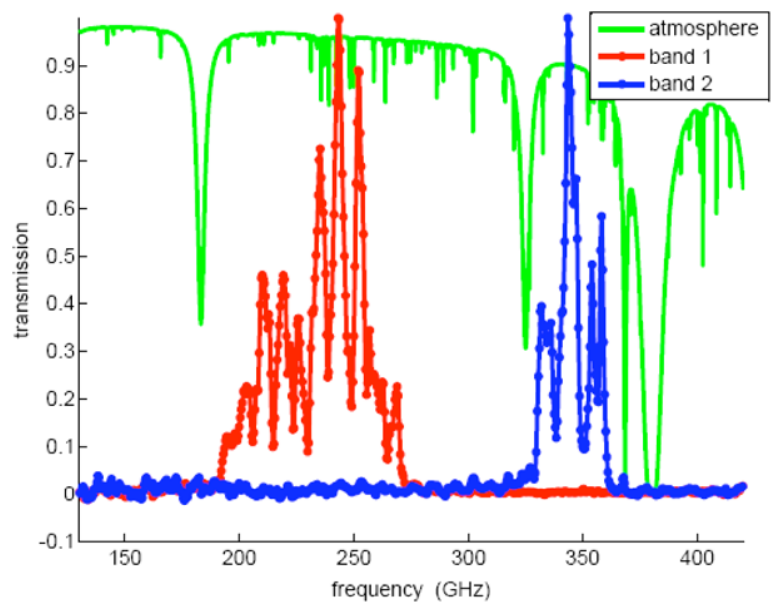

mirrors to focus the light from the Cassegrain focus of the antenna onto the focal plane. The cold optics consisted only of a single PTFE lens to achieve the desired plate scale. We avoided using metal mesh filters, instead opting for a purely dielectric filter stack comprised of PTFE and Fluorogold. All filters and the lens were antireflection coated with Zitex.

In order to read out multiple detectors simultaneously, a digital method had to be developed to replace the standard analog readout. A software defined radio solution was employed [3], in which up to four probe signals could be generated in a $50 \mathrm{MHz}$ bandwidth. The signals could be mixed up to any desired frequency band containing resonators to be read out. The resonators were read out at $50 \mathrm{~Hz}$, much slower than the device response time governed by the measured $7 \mu$ s quasiparticle lifetimes.

To maximize the knowledge of sky-subtraction ability and relative calibration gained by the run, a non-uniform frequency spacing was used. This was designed to give us the choice of reading out four detectors of one color, or two antennas in two colors simultaneously. In practice, the resonant frequencies could not be predicted to accuracy sufficient to allow this for reasons that are not yet fully understood. However, one case of two-pixel, two-color readout was used extensively through the observing run.

\section{Performance}

First light with MKID resonators came with a raster scan observation of Jupiter. This feat was accomplished almost immediately after beginning on-sky observation, and resulted in an extremely high significance detection. However, observation showed that the detectors were still largely sensitive to small changes in magnetic fields at the array, as the scans included linear drifts noticeable compared to the large signal from Jupiter. This effect is most likely due to trapped magnetic flux in the detectors. An azimuth/elevation linear fit was thus employed, which removed the magnetic field effects. All the observations were either raster scans, most effective in alleviating sky noise, or drift scans, which eliminates any magnetic field effects by leaving the cryostat stationary for each scan. Linear response was achieved under any amount 

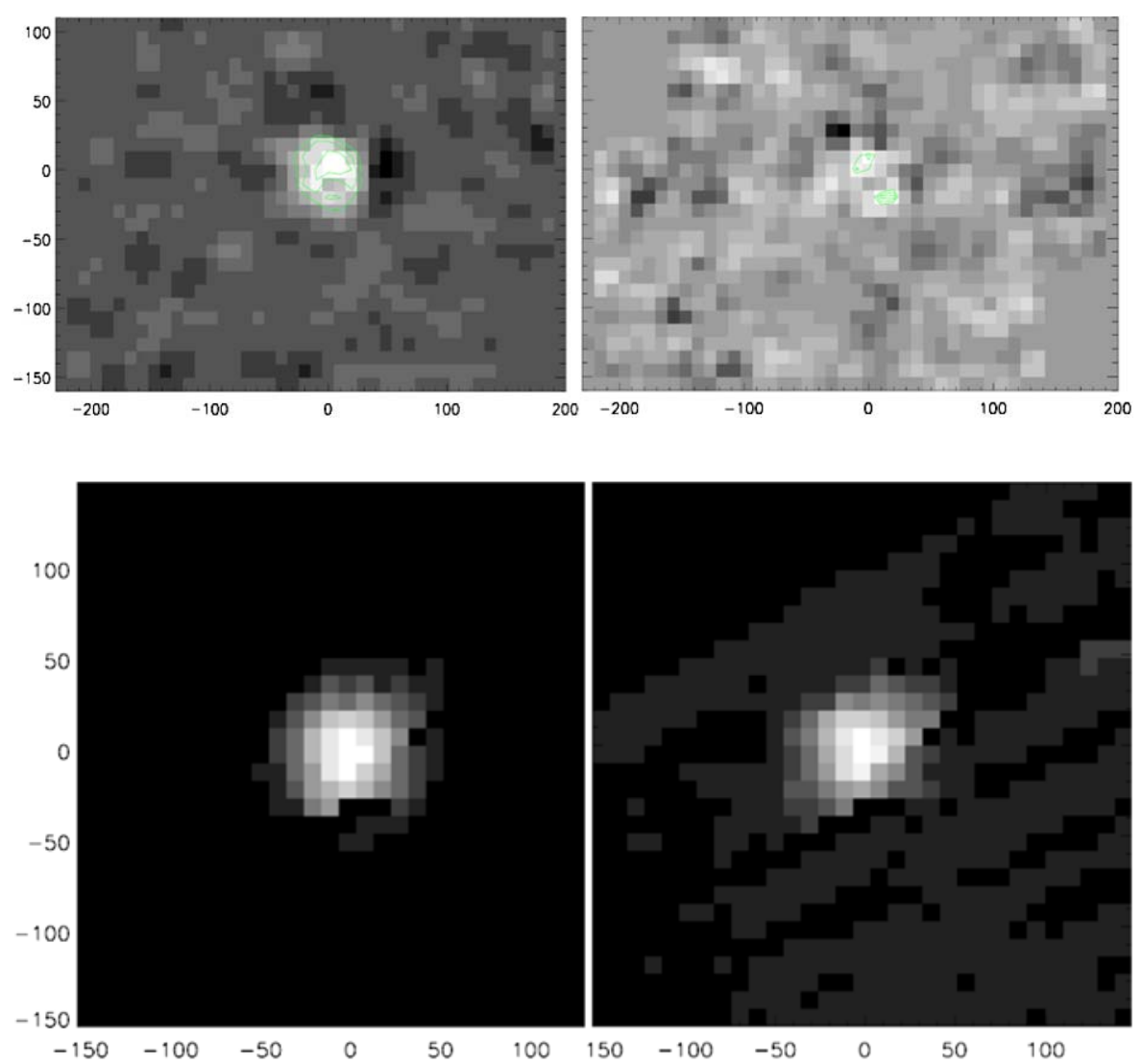

Fig. 2 Image of G34.3 from two pixels in two colors during the same observation. This observation involved drift scanning over the source for roughly 20 minutes, leading to a detection at $25 \sigma$ at $240 \mathrm{GHz}$

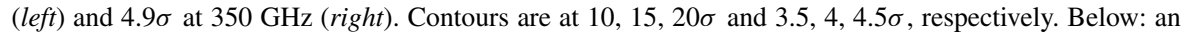
approximately 15 minute raster scan image of Jupiter at $240 \mathrm{GHz}$ and $350 \mathrm{GHz}$, each detected at $>1000 \sigma$. Map units are in arcseconds

of photon flux density from different atmospheric conditions by simply changing the probe frequency to match the resonance under load.

Given the two types of readout, amplitude and phase, for the detectors, we had two separate measures of the response. The response in the phase direction is found to be higher than the response in the amplitude direction, as expected. However, the phase response has much higher noise at low frequencies, while the amplitude noise is amplifier-limited [4]. At the low frequencies associated with telescope scan patterns, the amplitude signal yielded better signal-to-noise in the amplitude response for our observations.

We measured the system's Noise Equivalent Flux Density (NEFD) using numerous observations of planets and G34.3. We used the standard definition,

$$
\mathrm{NEFD}=\frac{F \sqrt{2 \Delta t}}{(S / N)}
$$


where $F$ is the source flux. Figure 2 shows a map of G34.3. Due to the fact that the planets were comparable to our beam size, we calculated the appropriate correction factors. We assumed a Gaussian beam, with width based on the FWHM of a diffraction pattern from the illuminated diameter of the antenna. We found that, for raster scans of planets, we achieved an NEFD of $1.3 \mathrm{Jy} \mathrm{s}^{1 / 2}$ at $240 \mathrm{GHz}$, and $8 \mathrm{Jy} \mathrm{s} \mathrm{s}^{1 / 2}$ at $350 \mathrm{GHz}$. In our observation of G34.3, we operated in drift scan mode, and thus expected to see more atmospheric 1/f noise. As expected, the noise was worse, yielding sensitivities of $1.6 \mathrm{Jy} \mathrm{s} \mathrm{s}^{1 / 2}$ and $14 \mathrm{Jy} \mathrm{s}{ }^{1 / 2}$, respectively. Further analysis of the data is required to determine the precise significance of the difference between scan strategies, and thus if the maps retain residual sky noise.

In images with two pixels and two colors, a two-color sky subtraction was employed, but the improvement was found to be negligible. This is likely due to the much worse responsivity of the $350 \mathrm{GHz}$ band and the comparable levels of detector and sky noise, and does not preclude significant sensitivity gains by using multicolor sky subtraction in future devices.

The detector noise performance at the CSO was comparable to noise data taken while observing a $300 \mathrm{~K}$ Eccosorb ${ }^{\mathrm{TM}}$ blackbody. Future noise data will be taken "dark", with no outward-looking window, to compare to noise at the telescope and find the true contribution from sky loading.

Although acceptable for an engineering run designed to simply demonstrate the concept, the sensitivities were roughly 20 times worse than the background limit of approximately $50 \mathrm{mJy} \mathrm{s}^{1 / 2}$ in the $240 \mathrm{GHz}$ band.

There are several reasons for the poorer-than-expected performance, as well as ways to remedy each. The dominant factor was that the optical efficiency was lower than expected by over an order of magnitude. In addition to the direct effect of the low efficiency, the deficiency of optical power on the detectors also decreases the detectors' coupling to the feedline and thereby reducing their responsivity. A higher optical efficiency would therefore improve the sensitivity in two ways. A new filter stack will be tested to reduce reflections and increase in-band transmission. Additional factors may include residual magnetic field signal and unexpected beam spillover. Magnetic field effects will be reduced in the future via magnetic shielding and by placing holes in the detector ground plane to pin magnetic flux that would otherwise trap in the MKID resonators.

\section{Future Plans}

The future MKID camera is designed to have 576 single-polarization antennas, each coupled to four resonators. The detectors will have in-line bandpass filters corresponding to the atmospheric windows at $240 \mathrm{GHz}, 270 \mathrm{GHz}, 350 \mathrm{GHz}$ and $400 \mathrm{GHz}$. However, the camera is designed such that a device substitution can be made to change the available passbands. A possible change would involve using lower frequencies (i.e. $150 \mathrm{GHz}$ ) for SZ cluster observations. The camera is funded and is expected to be deployed in 2010 .

The focal plane architecture will be adapted for the higher pixel count; rather than a single coaxial cable feeding the entire focal plane, eight separate coaxes will feed 
different octants, each amplified by its own HEMT cryogenic LNA. The reason for this is twofold. First, it is much easier to fabricate the devices in octants separately, assuring fewer technical challenges in uniformity across the wafer. Second, this allows for greater flexibility in the readout and amplifier noise characteristics. The noise in the digital readout and amplifier is power dependent, so reducing the amount of power through any element eases the process of reaching background limits.

As an intermediate step, we plan to use the DemoCam to develop and test our final arrays and readout electronics. Future changes will incorporate a $420 \mathrm{GHz}$ low-pass metal-mesh filter in the filter stack to further reduce out-of-band leakage, and obviate some dielectric filters. The next device will be designed to match optical loading such that the quality factor for coupling to the feedline, $Q_{c}$, matches the internal $Q$ due to quasiparticles in the superconductor. Such a device is critically coupled and will respond optimally to astronomical sources. The device will incorporate half of an octant of the final array, with 36 antennas feeding 4 resonators each, thus displaying the full multicolor ability of the antenna coupling. We will carry out an engineering run with the upgraded DemoCam in early 2008.

By this development towards a final camera, we will continue to develop MKIDs as highly sensitive, easily multiplexible arrays for applications in millimeter and submillimeter astronomy. This observing run showed the great promise of the detectors in future cameras, in spite of a number of resolvable technical difficulties.

Acknowledgements We are grateful to acknowledge the support of NASA (NNG06GG16G), the NSF (AST-0705157) and the generous contributions of the Gordon and Betty Moore Foundation.

\section{References}

1. P. Day et al., Nature 425, 817-821 (2003)

2. J. Glenn et al., Proc. SPIE 3357, 326-334 (1998)

3. B. Mazin et al., Nucl. Instrum. Methods Phys. 559(2), 799-801 (2006)

4. J. Gao et al., Appl. Phys. Lett. 90, 102507 (2007) 